\title{
Modeling and Measurements of CMUTs with Square Anisotropic Plates
}

la Cour, Mette Funding; Christiansen, Thomas Lehrmann; Dahl-Petersen, Christian; Reck, Kasper; Hansen, Ole; Jensen, Jørgen Arendt; Thomsen, Erik Vilain

\section{Published in:}

Proceedings of the 2013 IEEE International Ultrasonics Symposium

Link to article, DOI:

10.1109/ULTSYM.2013.0559

Publication date:

2013

Document Version

Early version, also known as pre-print

Link back to DTU Orbit

Citation (APA):

la Cour, M. F., Christiansen, T. L., Dahl-Petersen, C., Reck, K., Hansen, O., Jensen, J. A., \& Thomsen, E. V. (2013). Modeling and Measurements of CMUTs with Square Anisotropic Plates. In Proceedings of the 2013 IEEE International Ultrasonics Symposium (pp. 2187-2190). IEEE. https://doi.org/10.1109/ULTSYM.2013.0559

\section{General rights}

Copyright and moral rights for the publications made accessible in the public portal are retained by the authors and/or other copyright owners and it is a condition of accessing publications that users recognise and abide by the legal requirements associated with these rights.

- Users may download and print one copy of any publication from the public portal for the purpose of private study or research.

- You may not further distribute the material or use it for any profit-making activity or commercial gain

- You may freely distribute the URL identifying the publication in the public portal 


\title{
Modeling and Measurements of CMUTs with Square Anisotropic Plates
}

\author{
Mette Funding la Cour ${ }^{* \dagger}$, Thomas Lehrmann Christiansen ${ }^{\dagger}$, Christian Dahl-Petersen ${ }^{\dagger}$, Kasper Reck ${ }^{\dagger}$, \\ Ole Hansen ${ }^{\dagger}$, Jørgen Arendt Jensen* and Erik Vilain Thomsen ${ }^{\dagger}$ \\ *Center for Fast Ultrasound Imaging, Department of Electrical Engineering, Technical University of Denmark, \\ DK-2800 Kgs. Lyngby, Denmark \\ $\dagger$ Department of Micro and Nanotechnology, Technical University of Denmark, DK-2800 Kgs. Lyngby, Denmark
}

\begin{abstract}
The conventional method of modeling CMUTs use the isotropic plate equation to calculate the deflection, leading to deviations from FEM simulations including anisotropic effects of around $10 \%$ in center deflection. In this paper, the deflection is found for square plates using the full anisotropic plate equation and the Galerkin method. Utilizing the symmetry of the silicon crystal, a compact and accurate expression for the deflection can be obtained. The deviation from FEM in center deflection is $<0.1 \%$. The deflection was measured on fabricated CMUTs using a white light interferometer. Fitting the anisotropic calculated deflection to the measurement a deviation of $0.5-1.5 \%$ is seen for the fitted values. Finally it was also measured how the device behaved under increasing bias voltage and it is observed that the model including anisotropic effects is within the uncertainty interval of the measurements.
\end{abstract}

\section{INTRODUCTION}

Precise modeling of capacitive micromachined ultrasonic transducers (CMUT) is important for an efficient design process. The deflection $w(x, y)$ is an important parameter that influences several basic CMUT parameters such as pull-in voltage and capacitance. Most existing analytical approaches use the isotropic plate equation to calculate the deflection [1], [2]. However, when using fusion bonding fabrication technology the plate usually consists of crystalline silicon, which is an anisotropic material. The isotropic approach is then invalidated and this results in deviations in the deflection compared to finite element modeling (FEM) and measurements. Therefore, to get precise modeling of these CMUTs the anisotropy of silicon needs to be taken into account.

For circular plates a simple and exact solution for the deflection exists, but this is not the case for square plates. Existing solutions for the deflection of square plates is based on series expansions with either trigonometric [3] or polynomial basis functions [4]. None of these, however, take the anisotropy of the plate into account.

Previously a model was made for calculating the deflection for an anisotropic plate with circular geometry [5], and in this paper the model is expanded to include square plates as well. The approach used to solve the full anisotropic plate equation is the Galerkin method [6]. Utilizing the symmetry of the silicon crystal, a compact and accurate approximation of the deflection can be obtained. The calculated deflection is compared to the solution for corresponding isotropic cases, a finite element model (FEM) and measurements performed on fabricated devices. Furthermore, the calculated deflection is used to find the stable position of the CMUT plate for a given bias voltage. Equivalent measurements are performed as well and the theory is compared to these.

\section{THE ISOTROPIC PLATE EQUATION}

Conventionally the deflection $w(x, y)$ of a CMUT with a thin plate is modeled using the isotropic plate equation [3]

$$
\frac{\partial^{4} w}{\partial x^{4}}+2 \frac{\partial^{4} w}{\partial x^{2} \partial y^{2}}+\frac{\partial^{4} w}{\partial y^{4}}=\frac{p}{D_{\mathrm{i}}}
$$

where $p$ is the applied pressure difference across the plate. The flexural rigidity is given by

$$
D_{\mathrm{i}}=\frac{E}{12\left(1-v^{2}\right)} h^{3}
$$

with $E$ being Young's modulus, $v$ being Poisson's ratio, and $h$ being the thickness of the plate. For clamped rectangular and square plates no simple exact solution exists to this equation and approximate methods have to be used. The traditional isotropic approach is based on a series expansion of the deflection and the center deflection for a thin clamped square plate having side length $2 L$ is [3]

$$
w_{0, \text { isotropic }}=0.020245 \frac{L^{4} p}{D_{\mathrm{i}}} \text {. }
$$

However, the plate material is often not isotropic and (1) and (2) are therefore no longer valid. Using the fusion bonding fabrication technique the plate usually consist of silicon which is an anisotropic material with a diamond cubic crystal structure. Having a silicon (001) substrate, which are most often used, Young's modulus and Poisson's ratio are strongly anisotropic, and this leads to inaccurate deflection expressions.

\section{ANISOTROPIC PLATE EQUATION}

To be able to take the anisotropy of the plate into account and avoid the inaccuracy from isotropic modeling, the stiffness of the plate needs to be described through the stiffness matrix of the material instead of Young's modulus and Poisson's ratio. The starting point is the relation between stress and strain [7]

$$
\sigma^{\mathfrak{c}}=\mathbf{c}^{\mathfrak{c}} \varepsilon^{\mathfrak{c}} \text {, or } \varepsilon^{\mathfrak{c}}=\mathbf{s}^{\mathfrak{c}} \sigma^{\mathfrak{c}} .
$$

Here superscript $\mathrm{c}$ denotes the crystallographic coordinate system, so $\mathbf{c}^{\mathrm{c}}$ is the stiffness matrix and $\mathbf{s}^{\mathbf{c}}=\left(\mathbf{c}^{\mathrm{c}}\right)^{-1}$ the compliance matrix in this coordinate system. Having a thin plate the stresses in the $z$ direction can be ignored and plane 
TABLE I. ROOM TEMPERATURE (300K) COMPLIANCE COEFFICIENTS FOR LOW DOPED N-TYPE CRYSTALLINE SILICON [8].

\begin{tabular}{ccc}
\hline \hline \multicolumn{1}{c}{$s_{11}^{\mathrm{c}}$} & $s_{12}^{\mathrm{c}}$ \\
\hline $7.69 \times 10^{-12} \mathrm{~Pa}^{-1}$ & $-2.14 \times 10^{-12} \mathrm{~Pa}^{-1}$ & $12.58 \times 10^{-12} \mathrm{~Pa}^{-1}$ \\
\hline \hline
\end{tabular}

stress assumed. The relation between strain and stress then becomes

$$
\left(\begin{array}{l}
\varepsilon_{1} \\
\varepsilon_{2} \\
\varepsilon_{6}
\end{array}\right)=\left(\begin{array}{lll}
s_{11} & s_{12} & s_{16} \\
s_{12} & s_{22} & s_{26} \\
s_{16} & s_{26} & s_{66}
\end{array}\right)\left(\begin{array}{l}
\sigma_{1} \\
\sigma_{2} \\
\sigma_{6}
\end{array}\right)=\mathbf{S}_{\text {eff }}\left(\begin{array}{l}
\sigma_{1} \\
\sigma_{2} \\
\sigma_{6}
\end{array}\right)
$$

and we can define an effective stiffness matrix, $\mathbf{C}_{\text {eff }}=\left(\mathbf{S}_{\text {eff }}\right)^{-1}$. For silicon the effective compliance matrix becomes

$$
\mathbf{S}_{\mathrm{eff}}^{\mathrm{c}}=\left(\begin{array}{ccc}
s_{11}^{\mathrm{c}} & s_{12}^{\mathrm{c}} & 0 \\
s_{12}^{\mathrm{c}} & s_{11}^{\mathrm{c}} & 0 \\
0 & 0 & s_{44}^{\mathrm{c}}
\end{array}\right)
$$

The compliance elements in this matrix are known from measurements and shown in Table I [8]. It is noted that the elements in (6) are known in the crystallographic coordinate system. An equation describing plate deflection on the other hand is valid in the plate coordinate system which is not necessarily the same. To illustrate this further the crystallographic and the plate coordinate systems can be seen in Fig. 1. The solid coordinate system aligned to the $\langle 100\rangle$ directions is where the compliance values for silicon are known and the dashed system shows the rotated coordinate system for the plate where the compliance values needs to be calculated. Having silicon as plate material and performing standard cleanroom fabrication, the plate will usually be on a (001) substrate and aligned to the primary wafer flat. Flat alignment is to the $\langle 110\rangle$ direction and the plate coordinate system will be rotated $\psi=45^{\circ}$ and a transformation of the compliance matrix between the two coordinate systems is needed. The resulting effective stiffness matrix for the present case becomes (taking the inverse of the transformed compliance matrix) [9]

$$
\begin{aligned}
& C_{\mathrm{Si}(001),[110]}^{\mathrm{eff}}= \\
& \left(\begin{array}{ccc}
\frac{1}{s_{44}^{c}}+\frac{1}{2\left(s_{11}^{c}+s_{11}^{c}\right)} & \frac{1}{2\left(s_{11}^{c}+s_{12}^{c}\right)}-\frac{1}{s_{44}^{c}} & 0 \\
\frac{1}{2\left(s_{11}^{c}+s_{12}^{c}\right)}-\frac{1}{s_{44}^{c}} & \frac{1}{s_{44}^{c}}+\frac{1}{2\left(s_{11}^{c}+s_{12}^{c}\right)} & 0 \\
0 & 0 & \frac{1}{2 s_{11}^{c}-2 s_{12}^{c}}
\end{array}\right) .
\end{aligned}
$$

It is seen that the stiffness matrix now has an orthotropic symmetry.

Now having the effective stiffness matrix the generalized plate equation can be used. This is a differential equation for the deflection, $w(x, y)$, of a thin anisotropic plate exposed to a uniform load $p$ given by [10], [9]

$$
\frac{\partial^{4} w}{\partial x^{4}}+k_{1} \frac{\partial^{4} w}{\partial x^{3} \partial y}+k_{2} \frac{\partial^{4} w}{\partial x^{2} \partial y^{2}}+k_{3} \frac{\partial^{4} w}{\partial x \partial y^{3}}+k_{4} \frac{\partial^{4} w}{\partial y^{4}}=\frac{p}{D_{\mathrm{a}}} .
$$

The plate coefficients $k_{1}-k_{4}$ and the anisotropic flexural rigidity, $D_{\mathrm{a}}$, depend on the elastic constants of the plate material

$$
\begin{gathered}
k_{1}=\frac{4 C_{13}^{\text {eff }}}{C_{11}^{\text {eff }}} \quad k_{2}=\frac{2\left(C_{12}^{\text {eff }}+2\right. \text { eff }}{\left.C_{11}^{\text {eff }}\right)} \quad k_{3}=\frac{4 C_{23}^{\text {eff }}}{C_{11}^{\text {eff }}} \\
k_{4}=\frac{C_{22}^{\text {eff }}}{C_{11}^{\text {eff }}} \quad D_{\mathrm{a}}=\frac{1}{12} h^{3} C_{11}^{\text {eff }}
\end{gathered}
$$

where $C_{p q}^{\text {eff }}$ are elements in the effective stiffness matrix. Notice that the stiffness of the plate is no longer expressed through

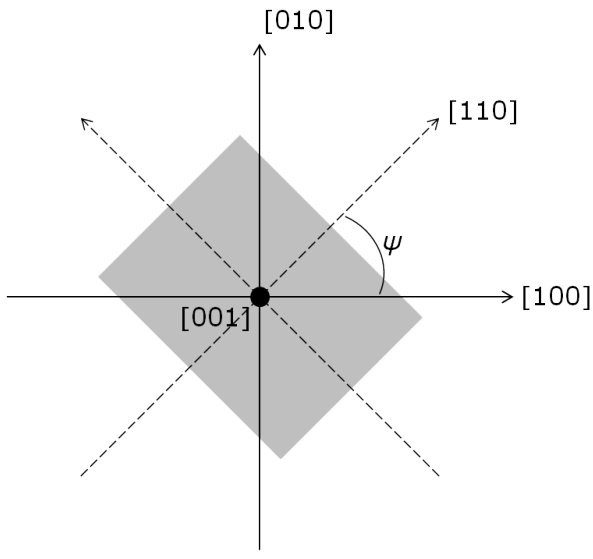

Fig. 1. The two coordinate systems, solid lines are the crystallographic system aligned to the $\langle 100\rangle$ direction and the dashed lines the plate system aligned to the $\langle 110\rangle$ direction.

TABLE II. SELECTED VAlUES FOR THE PLATE COEFFICIENTS AND ANISOTROPIC FLEXURAL RIGIDITY FOR PLATES ON A SILICON (001) SUBSTRATE [9].

\begin{tabular}{lcccccc}
\hline \hline Orientation & $\psi$ & $k_{1}$ & $k_{2}$ & $k_{3}$ & $k_{4}$ & $12 D_{a} / h^{3}[\mathrm{GPa}]$ \\
\hline$[100]$ & 0 & 0 & 2.8133 & 0 & 1 & 140.96 \\
{$[110]$} & $\pi / 4$ & 0 & 1.3241 & 0 & 1 & 169.62 \\
\hline \hline
\end{tabular}

Young's modulus and Poisson's ratio but directly through the stiffness values.

Using the compliance values for silicon in Table I and inserting the stiffness elements in (7) into (9) it follows that $k_{1}=k_{3}=0$ and $k_{4}=1$. Thus, aligning the plate to the primary flat simplifies the anisotropic plate equation (8) to

$$
\frac{\partial^{4} w}{\partial x^{4}}+k_{2} \frac{\partial^{4} w}{\partial x^{2} \partial y^{2}}+\frac{\partial^{4} w}{\partial y^{4}}=\frac{p}{D_{\mathrm{a}}} .
$$

The same is the case for aligning the plate along the [100] direction where the inverse of (6) is used instead of (7), giving the same values for $k_{1}, k_{3}$ and $k_{4}$. For these two special cases the coefficients in the plate equation are summarized in Table II.

\section{SOlVING THE Plate EQUATION}

Having a rectangular or square plate makes analytical deflection calculations complicated and approximate methods must be used to solve the generalized plate equation. With the anisotropic approach the Galerkin method [6] can be used to find approximate expressions for the deflection of a thin anisotropic square plate. In the most common case for CMUTs the plate is fabricated on a silicon (001) substrate and aligned to the [110] direction. For this orthotropic square plate with sidelengths $2 L$ the relative deflection is found to [9], [11]

$$
\begin{aligned}
\frac{w(x, y)}{w_{0}} & =\left[1-\left(\frac{x}{L}\right)^{2}\right]^{2}\left[1-\left(\frac{y}{L}\right)^{2}\right]^{2} \\
& \times\left[1+\beta\left(\frac{x}{L}\right)^{2}+\beta\left(\frac{y}{L}\right)^{2}\right],
\end{aligned}
$$

where the plate parameter is defined as

$$
\beta=\frac{182+143 k_{2}}{1432+91 k_{2}} \text {. }
$$


The center deflection can be written

$$
w_{0, \operatorname{Si}(001)}=\frac{77\left(1432+91 k_{2}\right)}{256\left(16220+11 k_{2}\left(329+13 k_{2}\right)\right)} \frac{L^{4} p}{D_{\mathrm{a}}} .
$$

Eqn. (11)-(13) are also valid when the plate is aligned to the [100] direction on a silicon (001) substrate. Note that the center deflection depends only on the $k_{2}$ coefficient. For primary flat alignment it is found by inserting $k_{2}$ into (12) that $\beta=0.23920$. This results in a normalized deflection surface for the plate aligned to the $\langle 110\rangle$ direction given by

$$
\begin{aligned}
\left.\frac{w(x, y)}{w_{0}}\right|_{\operatorname{Si}(001),\langle 110\rangle} & =\left[1-(x / L)^{2}\right]^{2}\left[1-(y / L)^{2}\right]^{2} \\
& \times\left[1+0.239207\left[(x / L)^{2}+(y / L)^{2}\right]\right]
\end{aligned}
$$

and the center deflection becomes

$$
\left.w_{0}\right|_{\operatorname{Si}(001),\langle 110\rangle}=0.02196 \frac{L^{4} p}{D_{\mathrm{a}}} .
$$

Comparing (3) and (15) it is seen that they are very similar containing the same parameters but different coefficients and the anisotropic instead of the isotropic flexural rigidity.

Fig. 2 shows the deflection cross section through $y=0$ of a square plate of silicon (001)

$$
w_{y=0}=w_{0}\left[1-(x / L)^{2}\right]^{2}\left[1+\beta(x / L)^{2}\right] .
$$

The deflection calculated with the anisotropic approach uses $k_{2}=1.3241$ in (12) and center deflection (15). This is compared to the isotropic approach using $k_{2}=2$ in (12) and center deflection (3), with Young's modulus and Poisson's ratio in the [100] and [110] directions, and to a finite element (FEM) simulation made using the full anisotropic compliance matrix (compliance coefficients from Table I) in COMSOL. The calculated deflections are normalized to the FEM center deflection. Excellent agreement is shown between the anisotropic curve and FEM with a deviation of less than $0.1 \%$ whereas the isotropic approach leads to deviations in the center deflection of around $10 \%$ for both [100] and [110] directions.

\section{CMUT APPLICATION}

Many important design parameters for CMUTs depend on the deflection of the plate. By using static analysis it is possible to find the stable position of the plate when applying a certain bias voltage. The stable position is easiest expressed through the center deflection and is the position where the strain force balance the electrostatic and pressure forces. The center deflection is found from energy considerations. The total potential energy of the system consists of three terms:

1) Strain energy. Calculated by integrating the strain energy density using (5), (7) and (11) and the result is

$$
\begin{aligned}
& U_{\mathrm{s}}=\frac{1}{2} \int_{-h / 2}^{h / 2} \int_{-L}^{L} \int_{-L}^{L}\left(\sigma_{1} \varepsilon_{1}+\sigma_{2} \varepsilon_{2}+\sigma_{6} \varepsilon_{6}\right) \mathrm{d} x \mathrm{~d} y \mathrm{~d} z \\
& U_{\mathrm{s}, \operatorname{Si}(001),[110]}=3.91172 \times 10^{11} \frac{h^{3} w_{0}^{2}}{L^{2}} .
\end{aligned}
$$

2) Energy due to applied pressure. This is found from the pressure load on the plate

$$
\begin{aligned}
& U_{\mathrm{p}}=-\int_{-L}^{L} \int_{-L}^{L} p w(x, y) d x d y \\
& U_{\mathrm{p}, \operatorname{Si}(001),[110]}=-1.216 p w_{0} L^{2} .
\end{aligned}
$$

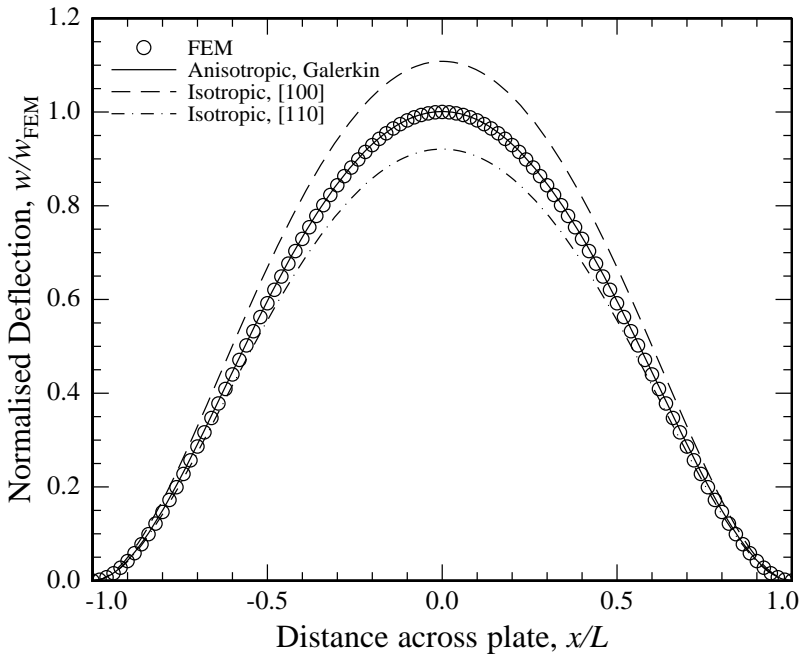

Fig. 2. Normalized deflection cross section $(y=0)$ of a square plate of silicon (001) calculated using both the isotropic approach with Young's modulus and Poisson's ratio in the [100] and [110] directions and the anisotropic approach. The circles represent the deflection calculated by FEM.

3) Electrostatic energy. Expressed through the charge $Q$ or applied voltage $V$, the vacuum permittivity $\varepsilon_{0}$, gap height $g$ and the total capacitance $C_{\mathrm{t}}$ of the device which for a square plate is found using a Taylor expansion of the integrant with the deflection in (11)

$$
\begin{aligned}
U_{\mathrm{e}} & =Q^{2} /\left(2 C_{\mathrm{t}}\right)=\frac{1}{2} V^{2} C_{\mathrm{t}} . \\
& =\frac{1}{2} V^{2} \int_{-L}^{L} \int_{-L}^{L} \frac{\varepsilon_{0}}{g-w(x, y)} d x d y
\end{aligned}
$$

The total force on the system is then found by differentiating the total potential energy with respect to the center deflection. From this the stable center position of the plate can be found for a given applied voltage as the point where the total force is zero.

\section{COMPARISON TO MEASUREMENTS}

CMUTs with square silicon plates have been fabricated using fusion bonding. The fabricated devices have a $65 \times 65$ $\mu \mathrm{m}$ wide and $2.37 \mu \mathrm{m}$ thick silicon plate with a gap height of $405 \mathrm{~nm}$ and a $198 \mathrm{~nm}$ thick insulating oxide at the bottom of the cavity. The deflection was measured with a Sensofar PLu Neox 3D Optical Profiler using white light interferometry.

Fig. 3 shows a measured cross section of the normalized deflection for the fabricated device. It is normalized in both center deflection and distance across the plate to compare the shape of the measured deflection with the calculated deflection. The red curve is a fit made to the measurements using the anisotropic model (16). Both the center deflection and the plate parameter $\beta$ is fitted. As it is seen in the figure the fitted value for $\beta$ is 0.243 which matches very well, with a deviation of $1.5 \%$, compared to the calculated value of 0.23920 for this type of plate (silicon (001) substrate aligned to [110] direction). The center deflection found from the fit has a deviation of $0.5 \%$ compared to the measurement. 


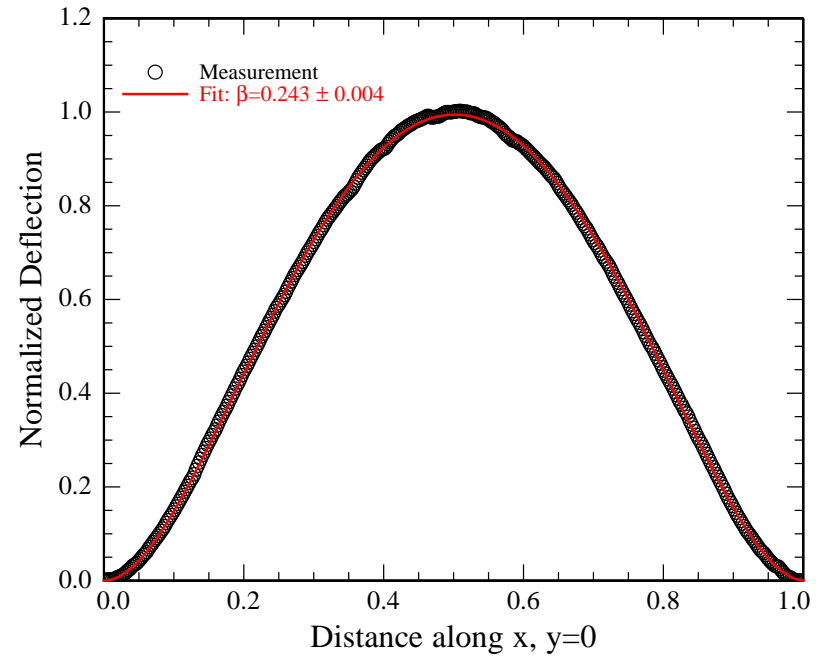

Fig. 3. Normalized deflection cross section from measurement on a fabricated CMUT. The red curve is a fit made with (16).

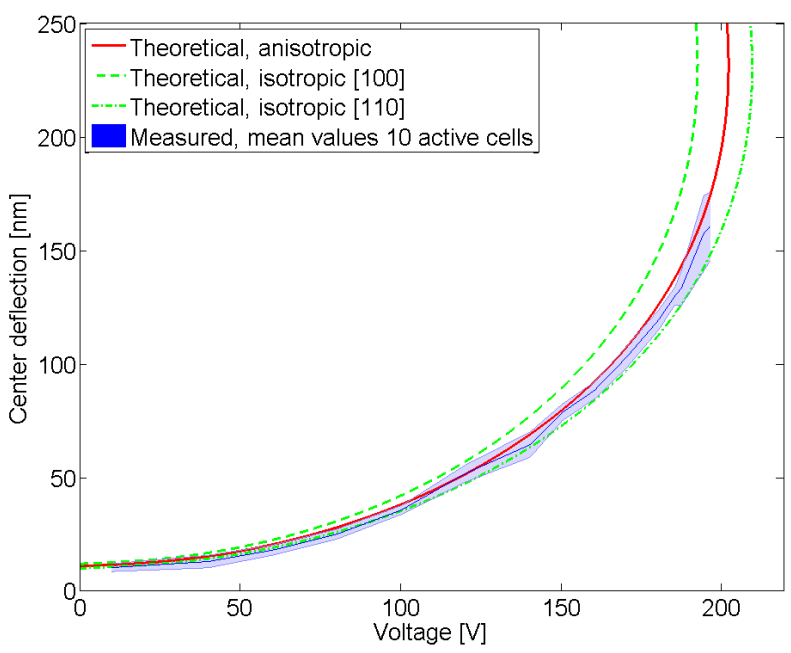

Fig. 4. Measured center deflection for increasing bias voltage together with theoretical curves using anisotropic and isotropic approaches.

Measurements with a DC voltage applied were also performed and the results are shown in Fig. 4. Here it is seen how the center deflection varies with the applied voltage and how it deflects more when approaching the pull-in voltage as expected. The center deflection for the measurements is found as the average of 10 cells. The errorbars corresponds to plus/minus two standard deviations. A theoretical curve made from the stable position analysis described in section $\mathrm{V}$ is plotted as well for both anisotropic and isotropic [100] and [110] approaches. The thickness of the plate is adjusted to $2.48,2.49$ and $2.47 \mu \mathrm{m}$ respectively with the correction found by FEM to take the $200 \mathrm{~nm} \mathrm{Al} \mathrm{layer} \mathrm{on} \mathrm{top} \mathrm{of} \mathrm{the} \mathrm{Si} \mathrm{into}$ account. It is seen that the anisotropic theory matches well with the measurement as it is within the error margin and the isotropic curves show similar behavior as in Fig. 2. Also the pull-in voltage is in good agreement as it was measured to be $206 \mathrm{~V}$, compared to an expected value of $202 \mathrm{~V}$ from the anisotropic model.

\section{CONCLUSION}

Using isotropic plate theory to calculate the deflection of anisotropic silicon plates results in deviations from FEM or measurements of up to $10 \%$. The full anisotropic plate equation was solved using the Galerkin method. It is seen that the deflection simplifies by utilizing the symmetry of the silicon crystal and a compact solution is obtained for square CMUT plates on a (001) silicon substrate aligned to the [110] direction. The maximum deviation is less than $0.1 \%$ compared to FEM. Furthermore, the deflection was measured on fabricated devices and fitting the anisotropic calculated deflection to the measurement a deviation of $0.5-1.5 \%$ is observed in the fitted parameters. The stable position for varying bias voltage was also found using the anisotropic theory and comparing this to measurements it is seen that the theory is within the uncertainty interval of the measurements.

\section{ACKNOWLEDGEMENT}

This work was financially supported by the Danish National Advanced Technology Foundation (024-2008-3) and (82-2012-4).

\section{REFERENCES}

[1] I. O. Wygant, M. Kupnik, and B. T. Khuri-Yakub, "Analytically calculating membrane displacement and the equivalent circuit model of a circular CMUT cell," in IEEE Ultrasonics Symposium, 2008. IUS 2008. IEEE, Nov. 2008, pp. 2111-2114.

[2] A. Lohfink and P.-C. Eccardt, "Linear and nonlinear equivalent circuit modeling of CMUTs," IEEE Transactions on Ultrasonics, Ferroelectrics and Frequency Control, vol. 52, no. 12, pp. 2163 -2172, Dec. 2005.

[3] R. L. Taylor and S. Govindjee, "Solution of clamped rectangular plate problems," Communications in Numerical Methods in Engineering, vol. 20, no. 10, p. 757765, 2004. [Online]. Available: http://onlinelibrary.wiley.com/doi/10.1002/cnm.652/abstract

[4] M. Rahman, J. Hernandez, and S. Chowdhury, "An improved analytical method to design CMUTs with square diaphragms," IEEE Transactions on Ultrasonics Ferroelectrics and Frequency Control, vol. 60, no. 4, pp. 834-845, Apr. 2013, WOS:000317010200020.

[5] M. F. la Cour, T. L. Christiansen, J. A. Jensen, and E. V. Thomsen, "Modelling of CMUTs with anisotropic plates," Proceedings of IEEE International Ultrasonics Symposium, pp. 588-591, 2012.

[6] E. Ventsel and T. Krauthammer, Thin Plates and Shells: Theory: Analysis, and Applications. Taylor \& Francis, Aug. 2001.

[7] R. E. Newnham, Properties of Materials: Anisotropy, Symmetry, Structure. Oxford University Press, USA, Jan. 2005.

[8] J. J. Hall, "Electronic effects in the elastic constants of n-type silicon," Physical Review, vol. 161, no. 3, pp. 756-761, Sep. 1967. [Online]. Available: http://link.aps.org/doi/10.1103/PhysRev.161.756

[9] E. V. Thomsen, K. Reck, G. Skands, C. Bertelsen, and O. Hansen, "Silicon as an anisotropic mechanical material: Deflection of thin crystalline plates," submitted to Journal of Microelectromechanical Systems.

[10] S. Holgate, "The transverse flexure of perforated aeolotropic plates," Proceedings of the Royal Society A: Mathematical, Physical and Engineering Sciences, vol. 185, no. 1000, pp. 50-69, Jan. 1946. [Online]. Available: http://adsabs.harvard.edu/abs/1946RSPSA.185...50H

[11] F. Mbakogu and M. Pavlovi, "Bending of clamped orthotropic rectangular plates: a variational symbolic solution," Computers \& Structures, vol. 77, no. 2, pp. 117-128, Jun. 2000. [Online]. Available: http://www.sciencedirect.com/science/article/pii/S0045794999002175 